\title{
Three-Dimensional Volume-Rendered Series Complements 2D Orthogonal Multidetector Computed Tomography in the Evaluation of Abnormal Spinal Curvature in Patients at a Major Cancer Center: A Retrospective Review
}

\author{
J. Matthew Debnam, Leena Ketonen, and Nandita Guha-Thakurta \\ Section of Neuroradiology, Department of Diagnostic Radiology, The University of Texas MD Anderson Cancer Center, \\ 1515 Holcombe Boulevard, Unit 370, Houston, TX 77030, USA \\ Correspondence should be addressed to J. Matthew Debnam, matthew.debnam@mdanderson.org
}

Received 16 August 2012; Accepted 26 September 2012

Academic Editors: D. H. Clements and C.-H. Lee

Copyright (C) 2012 J. Matthew Debnam et al. This is an open access article distributed under the Creative Commons Attribution License, which permits unrestricted use, distribution, and reproduction in any medium, provided the original work is properly cited.

Background. Abnormal spinal curvature is routinely assessed with plain radiographs, MDCT, and MRI. MDCT can provide two-dimensional (2-D) orthogonal as well as reconstructed three-dimensional volume-rendered (3-D VR) images of the spine, including the translucent display: a computer-generated image set that enables the visualization of surgical instrumentation through bony structures. We hypothesized that the 3-D VR series provides additional information beyond that of 2-D orthogonal MDCT in the evaluation of abnormal spinal curvature in patients evaluated at a major cancer center. Methods. The 3-D VR series, including the translucent display, was compared to 2-D orthogonal MDCT studies in patients with an abnormal spinal curvature greater than 25 degrees and scored as being not helpful (0) or helpful (1) in 3 categories: spinal curvature; bony definition; additional findings (mass lesions, fractures, and instrumentation). Results. In 38 of 48 (79.2\%) patients assessed, the 3-D VR series were scored as helpful in 63 of $144(43.8 \%)$ total possible categories (32 spinal curvature; 14 bony definition; 17 additional findings). Conclusion. Three-dimensional MDCT images, including the translucent display, are complementary to multiplanar 2-D orthogonal MCDT in the evaluation of abnormal spinal curvature in patients treated at a major cancer center.

\section{Background}

Abnormal spinal curvature may be idiopathic or secondary to dystrophic etiologies, such as congenital, traumatic, and malignant causes. Initial assessment and followup of patients with an abnormal spinal curvature have routinely been performed using plain radiographs [1]. To evaluate dystrophic features, magnetic resonance imaging (MRI) has also been utilized $[2,3]$. Computed tomography (CT) is proving to be of benefit in the assessment of patients with an abnormal spinal curvature [4-10]. Recent advances in multidetector CT (MDCT) technique allow the evaluation of the spine in multiple 2-D planes (Figures $1(\mathrm{a})$ and $2(\mathrm{a})$ ) and with a threedimensional volume-rendered series (3-D VR) (Figures 1(b) and 2(b)). In addition, the data from the MDCT study can also be used to generate a translucent display, a computergenerated image set that provides 3-D images of the spine enabling assessment of surgical instrumentation through the bony structures (Figures 2(c) and 2(d)). The 3-D VR series, including the translucent display, which are generated by computer manipulation of the axial CT source data without additional radiation, have led to a growth in demand for MDCT for the imaging of the spine by the spine surgeons at our institution, a major cancer center. However, to the best of our knowledge, there are no reports in the literature on the use of MDCT with the 3-D VR series and the translucent display for evaluation of abnormal spinal curvature, which requires additional time for processing and interpretation. We tested the hypothesis that the 3-D VR series, including the translucent display, provides additional information beyond 


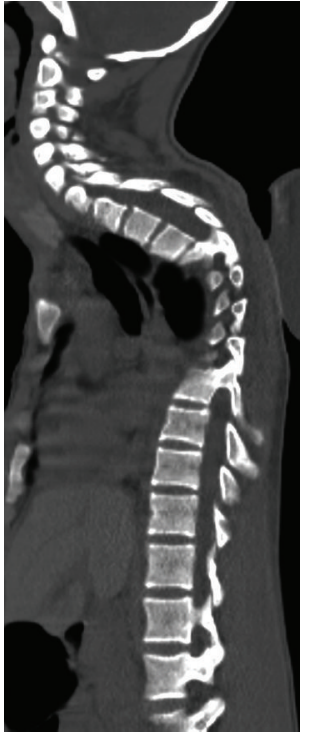

(a)

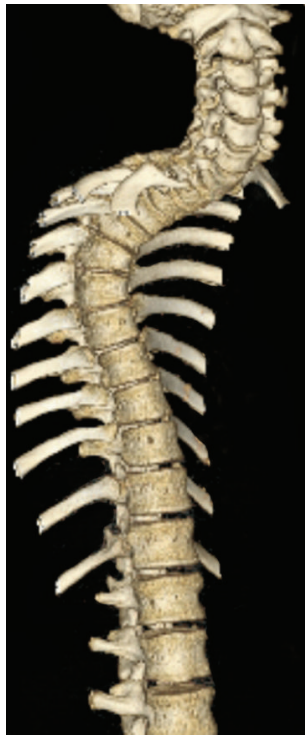

(b)
Figure 1: Patient 11, a 16-year-old male who underwent resection of an intramedullary juvenile pilocytic astrocytoma and presented with increasing thoracic kyphosis. (a) Sagittal MDCT image demonstrating the kyphotic deformity. Although consecutive images can be scrolled through the spine, each image is viewed singularly, limiting the evaluation of the abnormal curvature. (b) 3-D volume-rendered series, demonstrating kyphotic spinal curvature, can be rotated and viewed from $360^{\circ}$.

that of the 2-D orthogonal MDCT in the evaluation of abnormal spinal curvature in patients treated at a cancer center.

\section{Methods}

The Institutional Review Board of The University of Texas MD Anderson Cancer Center approved this study and waived the requirements for an informed consent. MDCT studies of the spine in patients who had a history of possible primary or metastatic disease to the spine or neurofibromatosis type 1 and an abnormal spinal curvature greater than 25 degrees, as assessed on MDCT, were included in our study. Measurements of spinal curvature were made on the 2-D coronal or sagittal reconstructed CT images, as described in the literature $[4,5]$. The review of the imaging studies and clinical data was performed by 3 neuroradiologists (JMD, LK, and NGT) in a consensus fashion. The 3D VR series, including translucent display, was compared to the 2-D orthogonal MDCT studies without the 3-D VR series in the assessment of the following 3 categories: spinal curvature; bony definition (deformity, fusion, and destruction); additional findings (mass lesions, fractures, and instrumentation). For each patient, the 3-D VR series was given a score of 1 if it was judged to be "helpful" in the evaluation of the aforementioned 3 categories (48 patients $\times$ 3 categories $=144$ total possible categories) and a score of 0 if it was "not helpful".
MDCT examinations were performed on a multidetector CT scanner (GE Medical Systems) to yield imaging in the axial plane using the following parameters: $140 \mathrm{kV}, 220$ $250 \mathrm{~mA}$, and a $1.25 \mathrm{~mm}$ slice thickness. The MDCT examinations were performed without $(n=41)$, with $(n=3)$, or without and with $(n=4)$ intravenous contrast (Optiray, Mallinckrodt Inc., St. Louis, MO, USA). Bone algorithm and soft tissue images were available and reviewed in all patients' MDCT studies. Postprocessing was then performed by a trained technologist on an advantage AW4.2 workstation (GE Medical Systems) using Volume View software (GE Medical Systems). The post-processing provided imaging in the sagittal and coronal planes in all patients, and these images, together with the axial source images, are hereafter referred to as the 2-D orthogonal MDCT study. In addition, 3-D VR images of the spine (Figures 1(b) and 2(b)) and the translucent display (Figures 2(c) and 2(d)), for patients in whom surgical instrumentation was placed for the stabilization of abnormal spinal curvature, were provided.

\section{Results}

The study included 48 patients (35 female and 13 male; ages 12-72, mean age, 48 years), as summarized in Table 1. Twenty-four patients had a dextroscoliotic curvature measuring between 26 and 93 degrees (mean, 45.5), 9 patients had a levoscoliotic curvature measuring between 25 and 72 degrees (mean, 35.2 degrees), and 15 patients had a kyphotic curvature of measuring between 27 and 89 degrees (mean 48.1 degrees). Twenty-six patients had a 3-D VR series without a transparent display and 22 patients had a 3-D VR series with a transparent display.

The 3-D VR series was rated as "helpful" when compared to the 2-D orthogonal MDCT study in 38 of $48(79.2 \%)$ patients; in 10 of $48(20.8 \%)$ patients, the 3-D VR series was rated as "not helpful". The 3-D VR series were scored as "helpful" in 63 of $144(43.8 \%)$ total possible categories (Table 2). This included the assessment of the spinal curvature in 32 of $48(66.7 \%)$ patients, including dextroscoliosis $\left(n=16,26-83^{\circ}\right.$, mean $\left.50.6^{\circ}\right)$, levoscoliosis $\left(n=8,25-72^{\circ}\right.$, mean $\left.35.3^{\circ}\right)$, and kyphosis $\left(n=8 ; 42-89^{\circ}\right.$, mean $\left.58.9^{\circ}\right)$. The 3 -D VR series was rated as "not helpful" in 16 of 48 patients (33.3\%), including those with dextroscoliosis $\left(n=8 ; 29-49^{\circ}\right.$, mean $\left.35.4^{\circ}\right)$, levoscoliosis $\left(n=1,35^{\circ}\right)$, and kyphosis $(n=7$; $27-47^{\circ}$, mean $35.7^{\circ}$ ).

The 3-D VR series was rated as "helpful" in the bone definition category for 14 of 48 (29.2\%) patients, including bone deformity $(n=6)$, bony fusion $(n=4)$, bony destruction $(n=2)$, anterolisthesis $(n=2)$, and the additional findings category, including surgical instrumentation, in 17 patients, specifically, for the assessment of the fusion rods $(n=15)$ or an anterior fusion plate and pedicular screws $(n=2)$.

\section{Illustrative Cases}

Figure 1 shows 2-D orthogonal MDCT and 3-D VR images from patient no. 11, a 16-year-old male who presented with a kyphotic curvature secondary to a recurrent juvenile 


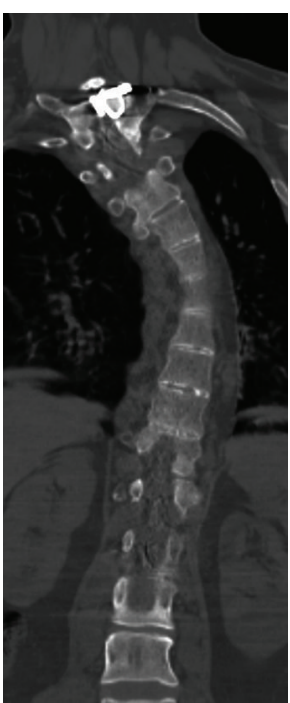

(a)

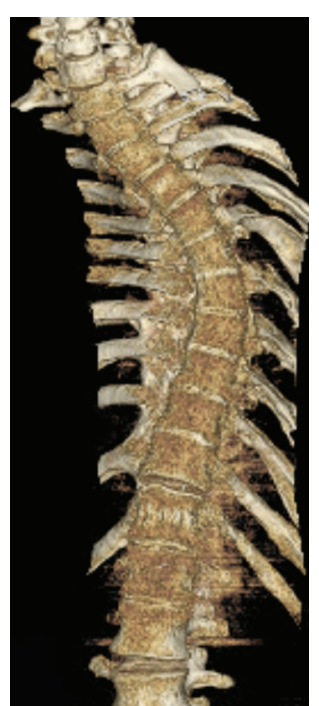

(b)

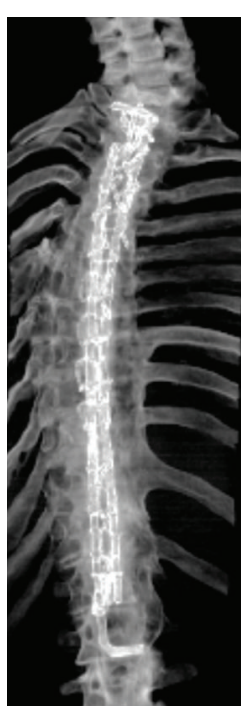

(c)

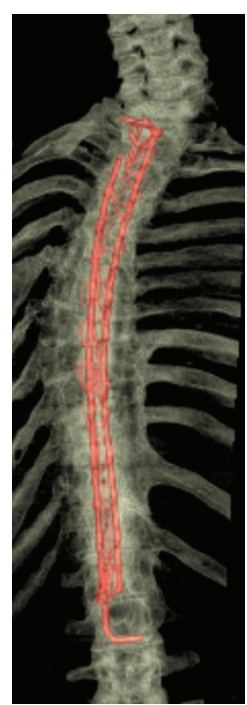

(d)

FIGURE 2: Patient 4, a 61-year-old woman with breast cancer who underwent correction of scoliosis with rod fixation at an outside institution. (a) 2-D coronal MDCT reconstruction demonstrating the scoliotic deformity and only a portion of the posterior instrumentation. (b) 3$\mathrm{D}$ volume-rendered series demonstrating the surface of the vertebral bodies in the scoliotic spine. (c) 3-D volume-rendered series with transparent display demonstrating the position and integrity of the spinal instrumentation. This series can be rotated and viewed from $360^{\circ}$. (d) Color-coded 3-D volume-rendered series with transparent display demonstrating the spinal instrumentation same image as (c), but color-coded to illustrate instrumentation.

pilocytic astrocytoma of the thoracic spinal cord. The 3-D VR images were scored as 1, "helpful" in the evaluation of the spinal curvature as the spine is out of plane on 2-D orthogonal MDCT imaging.

Figure 2 shows 2-D orthogonal MDCT and 3-D VR images from patient no. 4, a 61-year-old woman who underwent correction of an abnormal spinal curvature at an outside institution. The patient had a levoscoliotic curvature of the thoracic spine to such a degree the spinal column and instrumentation could not be visualized as one structure on a single, 2-D orthogonal MDCT image. The transparent display was helpful in demonstrating the scoliotic curvature and the position and integrity of the instrumentation.

\section{Discussion}

The 3-D VR series was rated as more "helpful" in comparison to the corresponding 2-D orthogonal MDCT in the assessment of abnormal spinal curvature in 38 of 48 patients, specifically in the evaluation of abnormal curvature in 32 patients, bony definition in 14 patients, and additional findings, including surgical instrumentation, in 17 patients. These findings confirm our hypothesis that the 3-D VR series, including the translucent display, is of additional benefit in the assessment of abnormal spinal curvature in patients treated at a major cancer center. As not all patients had abnormalities in each of the 3 categories, that is, surgical instrumentation, the percentage of cases where the 3-D VR was helpful is likely higher than what we report, further supporting our hypothesis.
When reviewing an MDCT study of abnormal spinal curvature, the 3-D VR series can be assessed before the 2$\mathrm{D}$ orthogonal MDCT study. As the spinal deformity in these patients is often out of the plane of imaging on a single, 2D orthogonal MDCT image (Figures 1(a) and 2(a)), the 3$D$ VR sequence provides comprehensive assessment of the entire spine on a single 3-D image; this image can then be rotated and viewed from 360 degrees. In our study, we found this more beneficial in patients with a greater degree of dextroscoliotic than kyphotic curvature and more helpful for a greater degree of abnormal spinal curvature. Not only can the 3-D VR series evaluate the shape of the spine, but also aid in the detection of a rotatory component, anterolisthesis, and the apex of the curvature. Any detected findings can then be confirmed on the 2-D MDCT study. In addition, with the 3 -D VR series, the number of vertebral bodies can be counted in a single view; this assures that the numbering assignment will be correct if an anomalous number of spinal segments are present. This is more difficult to determine on orthogonal 2-D MDCT as the spine is often out of the plane of imaging due to the spinal curvature.

The 3-D VR series can also evaluate the vertebral bodies for evidence of dysplasia, fracture, or bony destruction. Previous authors [10-12] have demonstrated that CT is better than plain radiographs for the evaluation of spine abnormalities. Kim et al. [10] state that when a complex osseous deformity is present, radiographs are inadequate for complete evaluation and the use of CT is mandatory, especially when surgery is planned. Our result takes this evaluation as a step further and demonstrate that the 3-D VR series would benefit 2-D orthogonal MDCT in the evaluation 
Table 1: Patient demographics.

\begin{tabular}{|c|c|c|c|c|}
\hline Patient no. & Age/sex & Reason for MDCT & Curvature (degrees) & Type \\
\hline 1 & $18 / \mathrm{F}$ & NF-1 & 81 & $\mathrm{~d}$ \\
\hline 2 & $32 / \mathrm{F}$ & NF-1 & 46 & $\mathrm{k}$ \\
\hline 3 & $63 / \mathrm{F}$ & NF-1 & 44 & $\mathrm{k}$ \\
\hline 4 & $61 / \mathrm{F}$ & Breast cancer & 30 & d \\
\hline 5 & $31 / \mathrm{F}$ & Undiagnosed paraspinal mass & 69 & d \\
\hline 6 & $38 / \mathrm{F}$ & NF-1 & 57 & $\mathrm{k}$ \\
\hline 7 & $36 / \mathrm{F}$ & NF-1 & 72 & 1 \\
\hline 8 & $44 / \mathrm{M}$ & NF-1 & 56 & $\mathrm{k}$ \\
\hline 9 & $15 / \mathrm{F}$ & Histiocytosis & 35 & d \\
\hline 10 & $63 / \mathrm{F}$ & Thyroid cancer & 61 & $\mathrm{k}$ \\
\hline 11 & $16 / \mathrm{M}$ & JPA & 76 & $\mathrm{k}$ \\
\hline 12 & $53 / \mathrm{F}$ & Breast cancer & 35 & d \\
\hline 13 & $12 / \mathrm{M}$ & NF-1 & 50 & d \\
\hline 14 & $42 / \mathrm{F}$ & NF-1 & 39 & d \\
\hline 15 & $20 / \mathrm{F}$ & NF-1 & 57 & d \\
\hline 16 & $19 / \mathrm{F}$ & NF-1 & 33 & d \\
\hline 17 & $19 / \mathrm{F}$ & NF-1 & 30 & d \\
\hline 18 & $29 / \mathrm{F}$ & NF-1 & 52 & d \\
\hline 19 & $65 / \mathrm{F}$ & Multiple myeloma & 42 & $\mathrm{k}$ \\
\hline 20 & $68 / \mathrm{F}$ & Pyriform sinus SCC & 29 & $\mathrm{k}$ \\
\hline 21 & $77 / \mathrm{M}$ & Lung cancer & 29 & $\mathrm{k}$ \\
\hline 22 & $54 / \mathrm{F}$ & Breast cancer & 35 & d \\
\hline 23 & $61 / \mathrm{M}$ & Multiple myeloma & 49 & d \\
\hline 24 & $21 / \mathrm{M}$ & Pelvic Ewings sarcoma & 40 & 1 \\
\hline 25 & $53 / \mathrm{M}$ & Thyroid cancer & 35 & 1 \\
\hline 26 & $66 / \mathrm{F}$ & Undifferentiated sarcoma of the spine & 42 & $\mathrm{k}$ \\
\hline 27 & $44 / \mathrm{F}$ & NF-1 & 54 & d \\
\hline 28 & $58 / \mathrm{F}$ & Breast cancer & 39 & $\mathrm{k}$ \\
\hline 29 & $56 / \mathrm{F}$ & NF-1 & 55 & d \\
\hline 30 & $32 / \mathrm{F}$ & NF-1 & 46 & d \\
\hline 31 & $82 / \mathrm{M}$ & Larynx chondrosarcoma & 47 & $\mathrm{k}$ \\
\hline 32 & $59 / \mathrm{F}$ & Met myxoid liposarcoma pelvis & 37 & $\mathrm{k}$ \\
\hline 33 & $61 / \mathrm{M}$ & Desmoid s/p laminectomy & 30 & 1 \\
\hline 34 & $77 / \mathrm{F}$ & Multiple myeloma & 27 & 1 \\
\hline 35 & $75 / \mathrm{M}$ & Prostate cancer & 26 & $\mathrm{~d}$ \\
\hline 36 & $30 / \mathrm{F}$ & NF-1 & 41 & d \\
\hline 37 & $39 / \mathrm{F}$ & NF-1 & 93 & d \\
\hline 38 & $40 / \mathrm{F}$ & Cervical cancer & 36 & 1 \\
\hline 39 & $62 / \mathrm{F}$ & Breast cancer & 27 & $\mathrm{k}$ \\
\hline 40 & $67 / \mathrm{F}$ & Thyroid cancer & 27 & 1 \\
\hline 41 & $71 / \mathrm{F}$ & $\mathrm{MFH}$ & 29 & d \\
\hline 42 & $56 / \mathrm{F}$ & NF-1 & 53 & d \\
\hline 43 & $55 / \mathrm{M}$ & Lymphoma & 34 & d \\
\hline 44 & $66 / \mathrm{F}$ & L3 plasmacytoma & 37 & d \\
\hline 45 & $33 / \mathrm{F}$ & Residual ependymoma & 25 & 1 \\
\hline 46 & $44 / \mathrm{F}$ & Lung cancer & 30 & d \\
\hline 47 & $71 / \mathrm{M}$ & Esophageal cancer & 89 & $\mathrm{k}$ \\
\hline 48 & $50 / \mathrm{M}$ & $\mathrm{HCC}$ & 25 & 1 \\
\hline
\end{tabular}

NF-1: neurofibromatosis type 1 ,

JPA: juvenile pilocytic astrocytoma,

SCC: squamous cell carcinoma,

MFH: malignant fibrous histiocytoma,

d: dextroscoliosis,

1: levoscoliosis,

k: kyphosis. 
TABLE 2: Categories-MDCT/3-D VR helpful to MDCT.

\begin{tabular}{|c|c|}
\hline Patient no. & Category \\
\hline 1 & Curvature, surgical fusion, and fusion rods \\
\hline 2 & Curvature, anterolisthesis \\
\hline 3 & Curvature \\
\hline 4 & Curvature, fusion rods \\
\hline 5 & Curvature, fusion rods \\
\hline 6 & Curvature, fusion rods \\
\hline 7 & Curvature, deformity, and fusion rods \\
\hline 8 & Curvature, fusion rods \\
\hline 10 & Curvature, anterolisthesis \\
\hline 11 & Curvature \\
\hline 12 & Destruction \\
\hline 13 & Curvature \\
\hline 14 & Curvature \\
\hline 15 & Curvature, deformity \\
\hline 16 & Fusion rods \\
\hline 17 & Fusion rods \\
\hline 18 & Curvature, fusion rods \\
\hline 20 & Bone plate/screws \\
\hline 23 & Pedicle screws \\
\hline 24 & Curvature, destruction \\
\hline 26 & Curvature, fusion rods \\
\hline 27 & Curvature, surgical fusion, and fusion rods \\
\hline 29 & Curvature, surgical fusion, and fusion rods \\
\hline 30 & Curvature, deformity \\
\hline 32 & Surgical fusion \\
\hline 33 & Curvature \\
\hline 34 & Curvature \\
\hline 35 & Curvature \\
\hline 36 & Curvature, deformity \\
\hline 37 & Curvature, deformity, and fusion rods \\
\hline 38 & Curvature \\
\hline 40 & Curvature \\
\hline 42 & Curvature, deformity, and fusion rods \\
\hline 43 & Curvature \\
\hline 45 & Curvature, fusion rods \\
\hline 46 & Curvature \\
\hline 47 & Curvature \\
\hline 48 & Curvature \\
\hline
\end{tabular}

of vertebral anomalies as the 14 studies scored a "helpful" rating in the evaluation of the bony structures, including 4 cases with prior surgical bony fusion.

In the post-operative patient, the 3-D VR series with translucent display may be used to assess surgical instrumentation [13]. This technique allows the visualization of the instrumentation through the bone and can also be rotated and viewed in 360 degrees, including in oblique planes. As the surgical instrumentation is visualized as one component on a single image, and the position and integrity of support rods and pedicular screws are assessed, coloring of the spinal instrumentation is also possible (Figure 2(d)). In every case with surgical instrumentation in our study, the 3-D VR with translucent display was rated as "helpful". In fact, our referring spine surgeons insist on inclusion of the 3-D VR series for the evaluation of the spine and the transparent display for a comprehensive overview of the surgical instrumentation. Further study can be undertaken to determine if the 3-D VR series with translucent display can be used to evaluate surgical instrumentation following spinal surgery in the general population with an abnormal spinal curvature and to evaluate associated complications.

As recent advances in MDCT technology have led to a significant reduction in streak artifact related to metallic hardware $[13,14]$. MDCT with VR series may lead to better evaluation of the postoperative spine and possibly easier detection of complications. It should be noted, though, that the interpreting radiologist must be careful not to mistake streak artifact that extends through the surgical instrumentation of the breakage of hardware.

The purpose of this study was to determine if the 3-D VR images provide additional information to the orthogonal 2D MDCT dataset and the results support our hypothesis. We are not suggesting that 3-D VR can replace the orthogonal 2D MDCT; rather 3-D VR is complementary. One limitation of the study is that this is a very select group of patients, mainly those presenting for treatment of their disease to a major cancer center.

Computed tomography has been described in the measurement of scoliosis, including the rotatory component [1519]. Further study will be necessary to determine if the 3-D VR series can be for evaluation and measurement of abnormal curvature in the general population, including for idiopathic scoliosis. One negative aspect of MDCT is that patients are imaged in the recumbent position and the use of ionizing radiation. The downside of patient positioning also applies to the reconstructed 3-D VR series; however, no addition radiation is necessary for computer generation of the 3-D VR series or the translucent display.

\section{Conclusion}

Herein, we have illustrated the added benefit of 3-D VR imaging and the translucent display to axial and 2-D orthogonal MDCT imaging of the spine for the evaluation of abnormal spinal curvature among patients at a major cancer center. Failure to recognize the etiology of spinal curvature, such as syndromic deformities, fractures, or malignancies of the spine, can affect treatment and management outcomes for patients. The translucent display series provides a more comprehensive evaluation of surgical instrumentation following correction of an abnormal spinal curvature or resection of malignancy. It is, therefore, beneficial for spine surgeons and radiologists involved in the care of patients with abnormal spinal curvature to be aware of the benefits of the 3-D VR series and the translucent display. 


\section{Abbreviations}

MRI: Magnetic resonance imaging

CT: $\quad$ Computed tomography

MDCT: Multidetector CT

2-D: Two-dimensional

3-D VR: Three-dimensional volumerendered.

\section{Acknowledgment}

The authors wish to thank Stephanie Deming for editorial assistance in the preparation of the paper.

\section{References}

[1] R. M. Slone, M. MacMillan, and W. J. Montgomery, "Spinal fixation. Part 3. Complications of spinal instrumentation.," Radiographics, vol. 13, no. 4, pp. 797-816, 1993.

[2] A. I. Tsirikos, M. Ramachandran, J. Lee, and A. Saifuddin, "Assessment of vertebral scalloping in neurofibromatosis type 1 with plain radiography and MRI," Clinical Radiology, vol. 59, no. 11, pp. 1009-1017, 2004.

[3] M. Cardoso and R. F. Keating, "Neurosurgical management of spinal dysraphism and neurogenic scoliosis," Spine, vol. 34, no. 17, pp. 1775-1782, 2009.

[4] C. J. Adam, M. T. Izatt, J. R. Harvey, and G. N. Askin, "Variability in Cobb angle measurements using reformatted computerized tomography scans," Spine, vol. 30, no. 14, pp. 1664-1669, 2005.

[5] J. Street, B. Lenehan, J. Albietz, P. Bishop, M. Dvorak, and C. Fisher, "Intraobserver and interobserver reliabilty of measures of kyphosis in thoracolumbar fractures," Spine Journal, vol. 9, no. 6, pp. 464-469, 2009.

[6] S. Göçen, H. Havitçioğlu, and E. Alici, "A new method to measure vertebral rotation from CT scans," European Spine Journal, vol. 8, no. 4, pp. 261-265, 1999.

[7] M. Krismer, W. Sterzinger, C. Haid, B. Frischhut, and R. Bauer, "Axial rotation measurement of scoliotic vertebrae by means of computed tomography scans," Spine, vol. 21, no. 5, pp. 576$581,1996$.

[8] M. Kamimura, T. Kinoshita, H. Itoh et al., "Preoperative CT examination for accurate and safe anterior spinal instrumentation surgery with endoscopic approach," Journal of Spinal Disorders, vol. 15, no. 1, pp. 47-51, 2002.

[9] R. M. Slone, K. W. McEnery, K. H. Bridwell, and W. J. Montgomery, "Principles and imaging of spinal instrumentation," Radiologic Clinics of North America, vol. 33, no. 2, pp. 189-211, 1995.

[10] H. Kim, H. S. Kim, E. S. Moon et al., "Scoliosis imaging: what radiologists should know," Radiographics, vol. 30, no. 7, pp. 1823-1842, 2010.

[11] P. O. Newton, G. W. Hahn, K. B. Fricka, and D. R. Wenger, "Utility of three-dimensional and multiplanar reformatted computed tomography for evaluation of pediatric congenital spine abnormalities," Spine, vol. 27, no. 8, pp. 844-850, 2002.

[12] D. J. Hedequist and J. B. Emans, "The correlation of preoperative three-dimensional computed tomography reconstructions with operative findings in congenital scoliosi," Spine, vol. 28, no. 22, pp. 2531-2534, 2003.

[13] L. M. R. Rodrigues, R. J. Nicolau, and C. Milani, "Computed tomographic evaluation of thoracic pedicle screw placement in idiopathic scoliosis," Journal of Pediatric Orthopaedics Part B, vol. 20, no. 4, pp. 195-198, 2011.
[14] K. A. Buckwalter, J. Rydberg, K. K. Kopecky, K. Crow, and E. L. Yang, "Musculoskeletal imaging with multislice CT," American Journal of Roentgenology, vol. 176, no. 4, pp. 979-986, 2001.

[15] A. K. Gupta, R. C. Nelson, G. A. Johnson, E. K. Paulson, D. M. Delong, and T. T. Yoshizumi, "Optimization of eight-element multi-detector row helical CT technology for evaluation of the abdomen," Radiology, vol. 227, no. 3, pp. 739-745, 2003.

[16] M. Yazici, E. R. Acaroglu, A. Alanay, V. Deviren, A. Cila, and A. Surat, "Measurement of vertebral rotation in standing versus supine position in adolescent idiopathic scoliosis," Journal of Pediatric Orthopaedics, vol. 21, no. 2, pp. 252-256, 2001.

[17] S. Göçen, M. G. Aksu, L. Baktiroğlu, and O. Ozcan, "Evaluation of computed tomographic methods to measure vertebral rotation in adolescent idiopathic scoliosis: an intraobserver and interobserver analysis," Journal of Spinal Disorders, vol. 11, no. 3, pp. 210-214, 1998.

[18] G. C. Lam, D. L. Hill, L. H. Le, J. V. Raso, and E. H. Lou, "Vertebral rotation measurement: a summary and comparison of common radiographic and CT methods," Scoliosis, vol. 3, no. 1, article no. 16, 2008.

[19] K. Abul-Kasim, M. K. Karlsson, R. Hasserius, and A. Ohlin, "Measurement of vertebral rotation in adolescent idiopathic scoliosis with low-dose CT in prone position-method description and reliability analysis," Scoliosis, vol. 5, no. 1, article no. $4,2010$. 


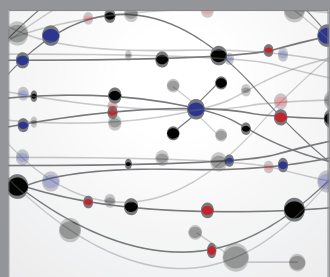

The Scientific World Journal
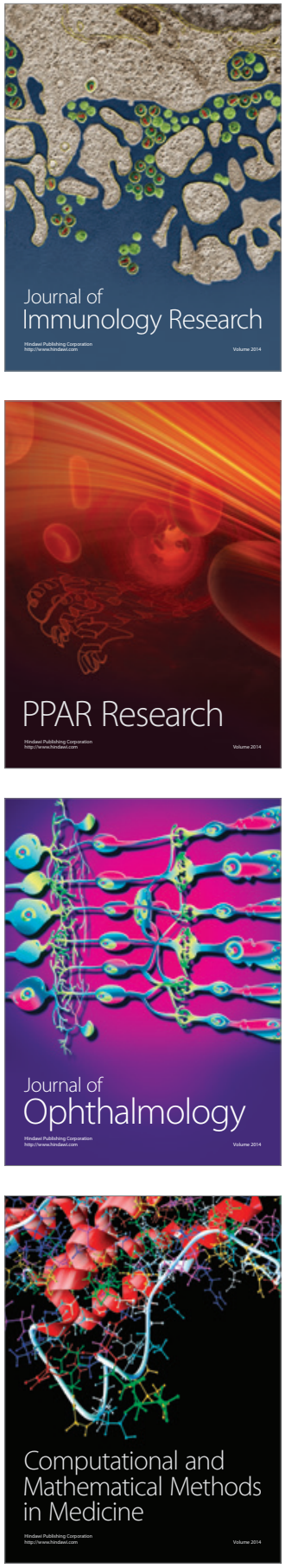

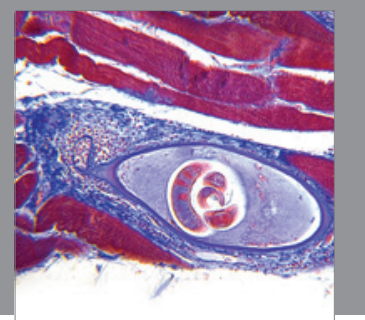

Gastroenterology

Research and Practice
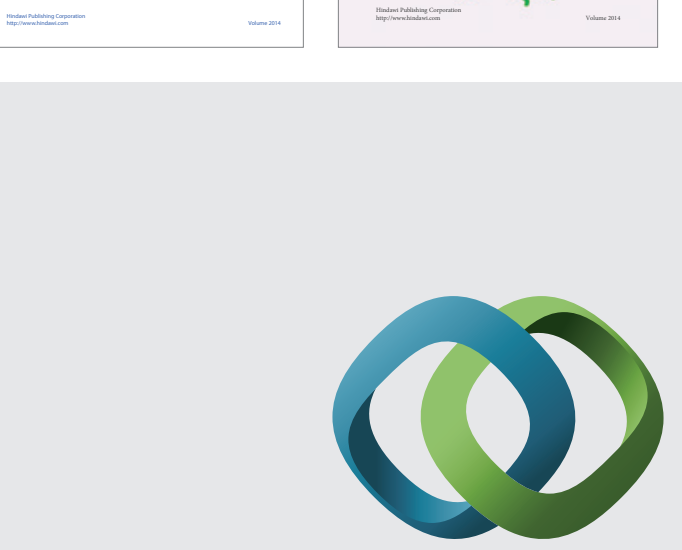

\section{Hindawi}

Submit your manuscripts at

http://www.hindawi.com
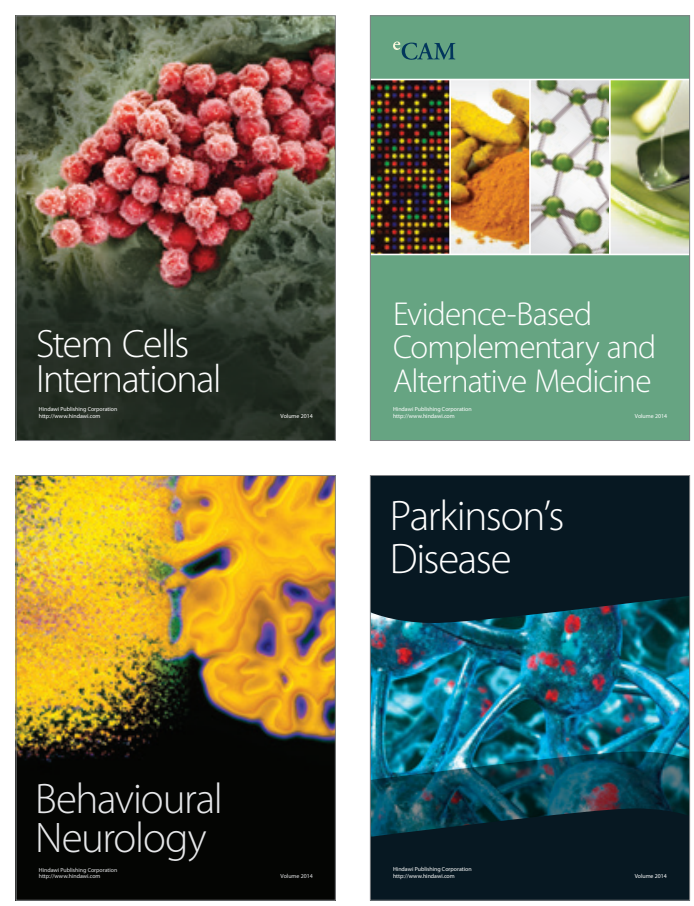

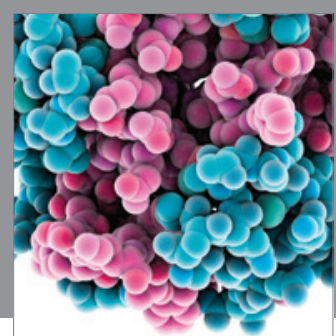

Journal of
Diabetes Research

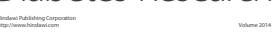

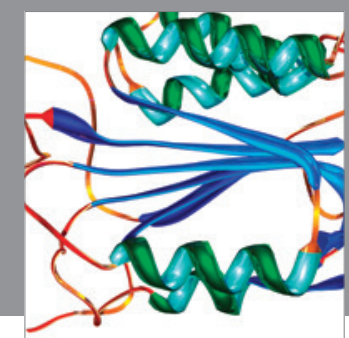

Disease Markers
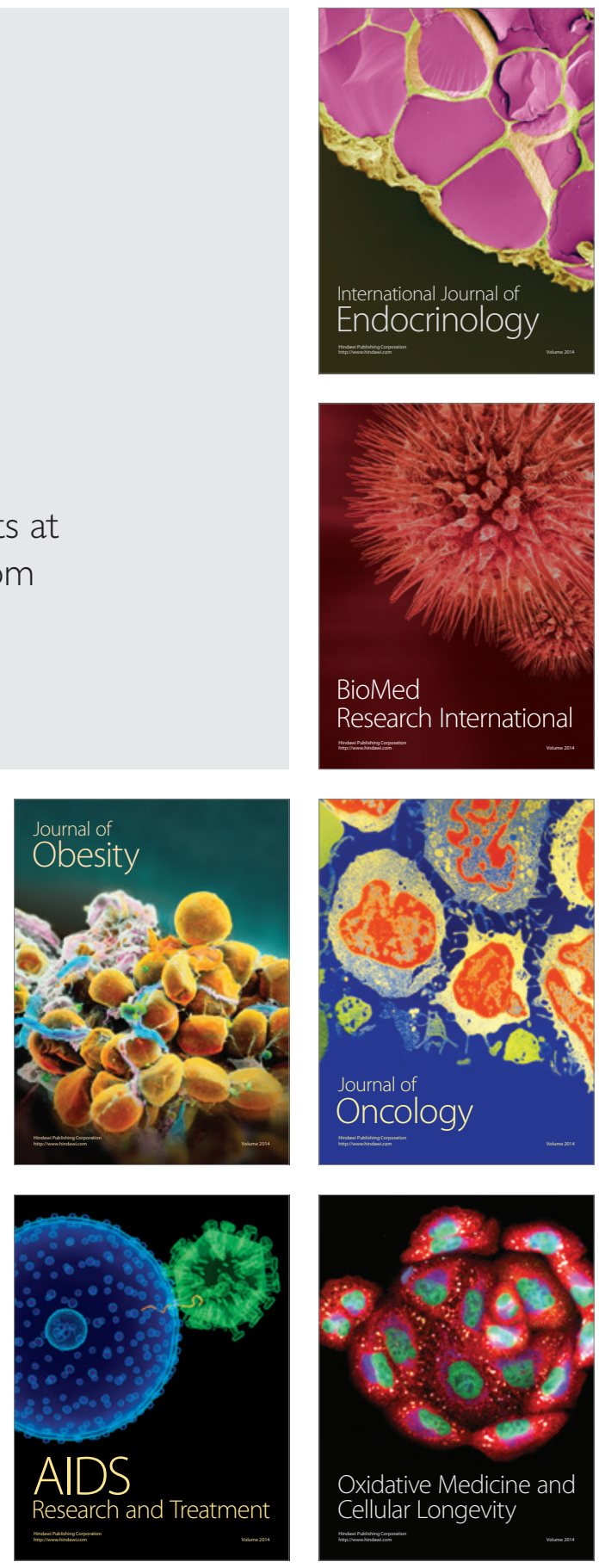